\title{
Causes behind error rates for predictive biomarker testing: the utility of sending post-EQA surveys
}

\author{
Cleo Keppens $^{1}$ (D) $\cdot$ Ed Schuuring $^{2}$ [D $\cdot$ Elisabeth M. C. Dequeker ${ }^{1}$
}

Received: 19 March 2020 / Revised: 29 October 2020 / Accepted: 1 November 2020 / Published online: 23 November 2020

(C) The Author(s) 2020

\begin{abstract}
External quality assessment (EQA) schemes assess the performance of predictive biomarker testing in lung and colorectal cancer and have previously demonstrated variable error rates. No information is currently available on the underlying causes of incorrect EQA results in the laboratories. Participants in EQA schemes by the European Society of Pathology between 2014 and 2018 for lung and colorectal cancer were contacted to complete a survey if they had at least one analysis error or test failure in the provided cases. Of the 791 surveys that were sent, 325 were completed including data from 185 unique laboratories on 514 incorrectly analyzed or failed cases. For the digital cases and immunohistochemistry, the majority of errors were interpretation-related. For fluorescence in situ hybridization, problems with the EQA materials were reported frequently. For variant analysis, the causes were mainly methodological for lung cancer but variable for colorectal cancer. Post-analytical (clerical and interpretation) errors were more likely detected after release of the EQA results compared to pre-analytical and analytical issues. Accredited laboratories encountered fewer reagent problems and more often responded to the survey. A recent change in test methodology resulted in method-related problems. Testing more samples annually introduced personnel errors and lead to a lower performance in future schemes. Participation to quality improvement projects is important to reduce deviating test results in laboratories, as the different error causes differently affect the test performance. EQA providers could benefit from requesting root cause analyses behind errors to offer even more tailored feedback, subschemes, and cases.
\end{abstract}

Keywords External quality assessment · Molecular pathology · Root cause analysis · Quality management · Biomarkers · ISO $15189 \cdot$ Colorectal cancer $\cdot$ Non-small-cell lung cancer

This article is part of the Topical Collection on Quality in Pathology

Supplementary Information The online version contains supplementary material available at https://doi.org/10.1007/s00428-02002966-7.

Elisabeth M. C. Dequeker

els.dequeker@kuleuven.be

Cleo Keppens

cleo.keppens@kuleuven.be

Ed Schuuring

e.schuuring@umcg.nl

1 Department of Public Health and Primary Care, Biomedical Quality Assurance Research Unit, University of Leuven, Kapucijnenvoer 35 block d, 1st floor, box 7001, 3000 Leuven, Belgium

2 Department of Pathology, University of Groningen, University Medical Center Groningen (UMCG), (HPC EA10), Hanzeplein 1, PO Box 30001, 9700 RB Groningen, The Netherlands

$\begin{array}{ll}\text { Abbreviations } \\ A L K & \text { ALK receptor tyrosine kinase } \\ B R A F & \text { B-Raf proto-oncogene } \\ \text { CAPA } & \text { Corrective/preventive action } \\ \text { CI } & \text { Confidence interval } \\ E G F R & \text { Epidermal growth factor receptor } \\ \text { EQA } & \text { External quality assessment } \\ \text { ESP } & \text { European Society of Pathology } \\ \text { FFPE } & \text { Formalin-fixed paraffin embedded } \\ \text { FISH } & \text { Fluorescence in situ hybridization } \\ \text { GEE } & \text { Generalized estimating equations } \\ \text { IHC } & \text { Immunohistochemistry } \\ \text { ISO } & \text { International Organization for Standardisation } \\ \text { KRAS } & \text { KRAS proto-oncogene } \\ \text { mCRC } & \text { Metastatic colorectal carcinoma } \\ \text { NGS } & \text { Next-generation sequencing } \\ \text { NRAS } & \text { NRAS proto-oncogene } \\ \text { NSCLC } & \text { Non-small-cell lung cancer }\end{array}$




$\begin{array}{ll}\text { OR } & \text { Odds ratio } \\ \text { PD-L1 } & \text { Programmed death ligand 1 } \\ \text { ROS1 } & \text { ROS proto-oncogene 1 } \\ \text { TPS } & \text { Tumor proportion score } \\ \text { TTP } & \text { Total test process } \\ \text { WT } & \text { Wild-type }\end{array}$

\section{Introduction}

The analysis of tumor-specific biomarkers provides information for appropriate targeted treatment decision-making in non-small-cell lung cancer (NSCLC) and metastatic colorectal cancer (mCRC) [1-3]. Predictive biomarker test results should therefore be accurate, reproducible and timely.

Several external quality assessment (EQA) schemes, organized on a national or international level, assessed the performance for common biomarkers in NSCLC and mCRC. They revealed varying error rates depending on the evaluated markers and variants, sample types, or scheme rounds [4-13].

Longitudinal analyses of the EQA schemes organized by the European Society of Pathology (ESP) revealed that participation to multiple EQA scheme rounds improved participants' performances $[12,13]$. Over time, error rates decreased for $A L K$ and EGFR analysis but increased for ROS1. Also, error rates were higher for immunohistochemistry (IHC) compared to fluorescence in situ hybridization (FISH) on formalin-fixed paraffin embedded (FFPE) samples and especially compared to digital case interpretation [12]. Remarkably, lower error rates have been described for cell lines compared to resections, for higher variant allele frequencies [13], and for laboratories who are accredited, test more samples or perform research [14]. In mCRC, error rates increased significantly for mutation-positive samples and for methods that do not cover all required variants [11].

Medical laboratories are advised to participate in EQA schemes $[1,3]$ sometimes part of their quality framework conform the International Organization for Standardization (ISO) standard 15189:2012 [15] or national equivalents like CAP 15189 [16]. Laboratories should have a documented procedure to identify and manage non-conformities when pre-determined performance criteria are not met, both for EQA as in routine practice.

The providers of these EQA programs are preferably accredited according to ISO 17043:2010 [17], mimic patient samples as closely as possible, and check the entire examination process [15]. EQA providers could guide laboratories by the provision of feedback, reference material, or methodological advice $[18,19]$. Some providers (such as the CAP and UK NEQAS) already request a root cause analysis from poor performers [7, 15], but no data has yet been published. Errors can be systematic (e.g., test method failure) while others can be accidental (e.g., clerical or pipetting errors). The time point of error occurrence in the total test process (TTP) has been reported in clinical chemistry and forensics $[20,21]$ and were mostly pre- (46-86\%) and post-analytical (18-47\%) of nature [20]. However, data is still lacking for molecular oncology.

Recently, a step-by-step framework for effective EQA results management was proposed for laboratories and EQA providers $[22,23]$. A subsequent evaluation of deviating EQA results in clinical chemistry according to this flowchart revealed that most errors ( $81 \%$ ) were the laboratory's responsibility (internal causes) and were mainly clerical errors (i.e., correct outcome entered incorrectly in the results form) (72\%) [22].

This study evaluated the feasibility of requesting root causes of deviating EQA results in the ESP schemes for NSCLC and mCRC between 2014 and 2018. The error causes were compared for the different markers, techniques, and sample types, as well as for different laboratory characteristics.

\section{Material and methods}

The ESP schemes were organized according to the requirements for EQA programs in molecular pathology [18] and ISO 17043 [17]. Laboratories could register to several subschemes for different techniques and markers. Sample selection and preparation, validation by the reference laboratories, and distribution to participants were previously described $[11,12]$. Laboratories received 14 calendar days to analyze all samples by their routine methodology and return an electronic datasheet on the cases' outcomes, the applied test methodology, and laboratory characteristics. Reported laboratory settings and accreditation statuses were further validated on the websites of the laboratories and national accreditation bodies, respectively. The correctness of the sample outcomes was assessed by a team of international experts according to predefined scoring criteria [11, 12] Participants received feedback including a general scheme report, participation certificate, and individual comments.

At the end of the EQA schemes, laboratories with at least one error or analysis failure (i.e., no outcome was available due to a test failure) were invited via e-mail to complete a survey with case-specific questions for every incorrect or failed case. The total number of participants and cases analyzed is summarized in Table 1. The survey was drafted in Microsoft Excel Developer and tailored to the participants' own results (Supplemental Data 1). This information included the case number and the type of deviation from the validated outcome for every subscheme (false-positive or false-negative results, variant reported at an incorrect position or gene, or over- and underestimations of the tumor proportion 
Table 1 Number of cases analyzed per subscheme offered in the ESP EQA schemes

\begin{tabular}{|c|c|c|c|c|c|c|c|}
\hline \multicolumn{3}{|l|}{ Year } & \multirow{2}{*}{2015} & \multirow{2}{*}{2016} & \multirow{2}{*}{2017} & \multirow{2}{*}{2018} & \multirow{2}{*}{$\frac{\text { Study total }}{2219}$} \\
\hline Analyzed laboratories & \multicolumn{2}{|c|}{ \# EQA participations to different subschemes } & & & & & \\
\hline & \multicolumn{2}{|c|}{ \# unique laboratories participating } & 197 & 234 & 259 & 241 & $410 *$ \\
\hline & \multicolumn{2}{|c|}{$\begin{array}{l}\text { \# unique laboratories who received the survey } \\
\text { (laboratories with at least one error in any of the } \\
\text { subschemes) }\end{array}$} & 88 & 120 & 153 & 175 & $315^{*}$ \\
\hline & \multicolumn{2}{|c|}{ \# unique laboratories who replied to the survey } & 39 & 44 & 90 & 99 & $185^{*}$ \\
\hline \multirow[t]{2}{*}{ Analyzed surveys } & \multicolumn{2}{|c|}{$\#$ of surveys sent } & 105 & 154 & 234 & 298 & 791 \\
\hline & \multicolumn{2}{|c|}{ \# of survey responses received } & 40 & 53 & 108 & 124 & 325 \\
\hline \multirow[t]{3}{*}{ Analyzed cases } & \multicolumn{2}{|c|}{ \# cases tested in the scheme } & 4224 & 5134 & 6276 & 5902 & 21,536 \\
\hline & \multicolumn{2}{|c|}{ \# deviating EQA results included in survey } & 162 & 225 & 362 & 418 & 1167 \\
\hline & \multicolumn{2}{|c|}{ \# deviating EQA results with response } & 51 & 74 & 181 & 208 & 514 \\
\hline \multirow[t]{14}{*}{ \# deviating EQA results with response for NSCLC } & \multirow[t]{2}{*}{ FISH digital } & $A L K$ & 1 & 0 & 4 & 2 & 7 \\
\hline & & ROS1 & 4 & 3 & 5 & 21 & 33 \\
\hline & IHC digital & PD-L1 & $\mathrm{N} / \mathrm{A}$ & N/A & 11 & 19 & 30 \\
\hline & \multirow[t]{2}{*}{ FISH } & $A L K$ & 2 & 4 & 18 & 1 & 25 \\
\hline & & ROS1 & 6 & 3 & 7 & 33 & 49 \\
\hline & \multirow[t]{3}{*}{$\mathrm{IHC}$} & ALK & 0 & 4 & 20 & 7 & 31 \\
\hline & & ROS1 & 9 & 0 & 2 & 6 & 17 \\
\hline & & PD-L1 & N/A & N/A & 35 & 28 & 63 \\
\hline & \multirow[t]{3}{*}{ IHC technical } & ALK & 8 & 6 & 7 & 15 & 36 \\
\hline & & ROS1 & N/A & 1 & 0 & 2 & 3 \\
\hline & & PD-L1 & N/A & N/A & N/A & 6 & 6 \\
\hline & \multirow[t]{3}{*}{ Variant analysis } & $E G F R$ (mandatory) & 21 & 19 & 41 & 27 & 108 \\
\hline & & $K R A S$ (optional) & N/A & N/A & 2 & 11 & 13 \\
\hline & & $B R A F$ (optional) & N/A & N/A & 0 & 3 & 3 \\
\hline \multirow[t]{3}{*}{ \# deviating EQA results with response for mCRC } & \multirow[t]{3}{*}{ Variant analysis** } & $K R A S$ (mandatory) & N/A & 23 & 23 & 22 & 68 \\
\hline & & NRAS (mandatory) & N/A & 7 & 2 & 3 & 12 \\
\hline & & $B R A F$ (optional) & N/A & 4 & 4 & 2 & 10 \\
\hline
\end{tabular}

Laboratories were free to participate to one of the techniques for a selected marker. Participation to FISH digital was mandatory for the same marker if a laboratory registered for FISH for that marker, and participation to IHC digital or technical was mandatory for IHC participants for the same marker. *One unique laboratory could have participated, received the survey, and replied to the survey in several scheme years, which is why the total number of unique participants does not equal the sum of the different years. **WT cases and cases without neoplastic cells are included within the schemes for $K R A S, N R A S$, and $B R A F$ to test the performance of the laboratories to denote these samples as a wild-type status or case without neoplastic cells

$N / A$, not applicable as no surveys were sent (no EQA scheme offered or only a pilot, or scheme outside the study period); $A L K$, ALK receptor tyrosine kinase; $B R A F$, B-Raf proto-oncogene; $E G F R$, epidermal growth factor receptor; $E Q A$, external quality assessment; $E S P$, European Society of Pathology; $F I S H$, fluorescence in situ hybridization; IHC, immunohistochemistry; KRAS, KRAS proto-oncogene; $m C R C$, metastatic colorectal cancer; $N R A S$, NRAS proto-oncogene; NSCLC, non-small cell lung cancer; $P D$ - L1, programmed death ligand 1; ROS1, ROS proto-oncogene 1

score (TPS) for $P D-L 1$ ). Questions included predeveloped dropdown lists and checkboxes for ease of completion.

Laboratories received additional information on the study set-up and a list of definitions on the applied terminology to harmonize responses for statistical analysis. The returned survey data were thereafter linked to the datasheet entries on laboratory setting and methodology during the EQA scheme, and the participants' performances. The deadline for response was set at 1 month.
Laboratories received a first reminder after 14 days and a second reminder the day before the deadline.

All survey responses from the ESP schemes for NSCLC between 2014 and 2018 and mCRC schemes between 2015 and 2018 were included. Statistics were performed using SAS software (version 9.4 of the SAS System for Windows, SAS Institute Inc., Cary, NC, USA). Statistical models with estimations using generalized estimating equations (GEE) were applied for clustering of identical laboratories participating to different schemes (NSCLC vs. mCRC) and years. Binary outcome variables were 
analyzed by logistic regression models. Ordinal and categorical outcome variables were analyzed by proportional odds models. Detailed statistics are shown in Supplemental Data 2.

\section{Results}

\section{Response to root cause surveys}

In the period between December 2015 and February 2019, 791 individual surveys were sent, to 315 unique laboratories from 43 countries. The probability of laboratories to receive the survey at the end of the EQA scheme (because they made an EQA error) and to respond to the survey is presented in Table 2 for the different laboratory characteristics.

Laboratories accredited conform ISO 15189 were less likely to receive the survey, as well as laboratories testing a larger number of annual samples for ROS1, KRAS, or NRAS, but not for the other markers. On the contrary, laboratories $(n=45)$ who outsourced a part of their analysis were more probable to receive the survey. Exact $p$ values and corresponding odds ratios (ORs) are shown in Supplemental Data 2A. Of the 45 respondents mentioning that they outsourced a part of the analysis, 15 outsourced the variant analysis itself, 6 outsourced both the DNA extraction and variant analysis, and 24 sent the samples to another laboratory for pathology review. There was no difference in the chance to receive the survey based on the laboratory's setting (university or community hospital), or number of personnel (Table 2).

Of the 791 surveys that were sent, $325(39.8 \%)$ responses were received by 185 unique laboratories $(58.4 \%)$ from 34 countries (Table 1). On average, the responses were received within 22.5 days (min. 1, max. 211, median 15 days). 139/325 (42.8\%) responses were received within the first 2 weeks (no reminder sent), 116 (35.7\%) after the first reminder, and 70 (21.5\%) after two reminders. The response time or number of reminders sent was not related to the laboratory characteristics (Supplemental Data 2A).

Accredited laboratories were more likely to return the completed survey compared to not accredited laboratories. Other factors did not influence the likelihood of responding to the received survey (Table 2).

\section{Time point in the total test process and cause of deviating EQA results}

Of the 988 NSCLC and $179 \mathrm{mCRC}$ cases with a deviating EQA result between 2015 and 2018, data was obtained for 424 (42.9\%) NSCLC and 90 (50.3\%) mCRC cases.

For the NSCLC EQA schemes $(n=424)$, errors occurred mostly in the post-analytical (48.1\%) phase (Table 3). For the digital cases, the majority of problems occurred in the post- analytical phase, given that these cases only comprised interpretation of pre-made images. This with the exception of some laboratories who implied a problem during the pre-analytical or analytical phase, when the images were created. For analysis of the FFPE samples, mainly post-analytical errors were observed for FISH and IHC, except for ALK FISH with $44.0 \%(n=25)$ analytical issues. During the IHC technical assessment, the staining quality of the applied protocol was evaluated, which is reflected in a high percentage of analytical issues as contributing factors for problems. For variant analysis, causes were mostly post-analytical for $E G F R$ testing $(47.2 \%, n=108)$ but analytical for $\operatorname{KRAS}(53.8 \%, n=13)$ and $B R A F(100.0 \%, n=3)$ testing. In the mCRC EQA schemes, all cases were tested by variant analysis, and results $(n=90)$ revealed mainly issues during the analytical phase itself (42.2\%), but percentages varied depending on the marker of analysis.

Analyzing the underlying causes (Table 4), both interpretation of the digital cases and IHC of the FFPE samples were prone to interpretation errors. For FISH analysis of FFPE cases, problems with the provided EQA material were most often reported. During the technical assessment, problems with the reagents were detected for ALK IHC, versus methodological problems for ROS1 IHC. For PD-L1, reasons of suboptimal staining quality were dispersed. For variant analysis in NSCLC, methodological issues were the main sources of errors, while for variant analysis in $\mathrm{mCRC}$, the underlying causes varied also depending on the analyzed marker.

The time point in the TTP and cause of the problems differed significantly between the indication (NSCLC vs. $\mathrm{mCRC}$ ), markers tested and techniques used (Supplemental Data 2B).

Definitions for the different categories and a more detailed cause of problems are given in Supplemental Data 3. Of all interpretation issues, 135 of 144 were reported in the NSCLC schemes. Of these, 51 (37.8\%) were reported during interpretation of the IHC staining intensity, 40 (29.6\%) during counting of the positive FISH signals, and to a lesser extent (18.5\%) due to an incorrect analysis of PCR curves during variant analysis. Causes for methodological problems reported in both schemes $(n=105)$ occurred mostly because the laboratories were unaware that the variant tested in the scheme was not included in their analysis method (35.2\%) or the method had an insufficient sensitivity to detect the variant at its respective frequency $(20.0 \%)$.

\section{Error causes for the different laboratory characteristics}

The probability to encounter a specific error cause in one of the phases of the TTP related to the laboratory characteristics as collected in the EQA datasheets is given in Table 5.

Pathology laboratories were significantly less probable of making a mistake in the pre-analytical phase and to denote the 
Table 2 Probability of survey receipt and response for the different laboratory characteristics

\begin{tabular}{|c|c|c|c|}
\hline Laboratory characteristics & $N$ & $\begin{array}{l}\text { Number of laboratories who received the survey } \\
\text { (min } 1 \text { error in EQA scheme) }(\%)\end{array}$ & $\begin{array}{l}\text { Number of laboratories who responded } \\
\text { (\% of participants receiving the survey) }\end{array}$ \\
\hline Setting $(n=2219)^{\mathrm{a}}$ & 2219 & $791(35.6)$ & $325(41.1)$ \\
\hline Industry & 71 & $33(46.5)$ & $15(45.5)$ \\
\hline (Private) laboratories & 399 & $151(37.8)$ & $64(42.4)$ \\
\hline Hospital laboratories & 585 & $217(37.1)$ & $100(46.1)$ \\
\hline University and/or research & 1164 & $390(33.6)$ & $146(37.4)$ \\
\hline Analysis under dept. of pathology $(n=2161)^{\mathrm{b}}$ & 2161 & $769(35.6)$ & $320(41.6)$ \\
\hline Yes & 1869 & $651(34.8)$ & $264(40.6)$ \\
\hline No & 292 & $118(40.4)$ & $56(47.5)$ \\
\hline Accreditation $(n=1874)^{\mathrm{c}}$ & 1874 & $702(37.5)^{*}$ & $285(40.6)^{*}$ \\
\hline Accredited & 861 & $306(35.5)$ & $139(45.4)$ \\
\hline Not accredited & 1013 & $396(39.1)$ & $146(36.9)$ \\
\hline Part of the analysis outsourced? $(n=340)^{\mathrm{d}}$ & 340 & $135(13.2)^{*}$ & $57(42.2)$ \\
\hline Yes & 45 & $24(53.3)$ & $10(41.7)$ \\
\hline No & 295 & $111(37.6)$ & $47(42.3)$ \\
\hline $\mathrm{Nr}$ of $\operatorname{staff}(n=2050)^{\mathrm{e}}$ & 2050 & $733(35.8)$ & $305(41.6)$ \\
\hline $1-5$ & 1002 & $353(35.2)$ & $146(41.4)$ \\
\hline $6-10$ & 638 & $234(36.7)$ & $83(35.5)$ \\
\hline $11-20$ & 284 & $101(35.6)$ & $58(57.4)$ \\
\hline$>20$ & 126 & $45(35.7)$ & $18(40.0)$ \\
\hline Number of EGFR samples tested last year? $(n=209)^{\mathrm{e}}$ & 209 & $80(38.3)$ & $32(40.0)$ \\
\hline No clinical samples tested & 8 & $5(62.5)$ & $2(40.0)$ \\
\hline$<10$ & 2 & $1(50.0)$ & $1(100.0)$ \\
\hline $10-99$ & 43 & $16(37.2)$ & $4(25.0)$ \\
\hline $100-249$ & 65 & $29(44.6)$ & $13(44.8)$ \\
\hline $250-499$ & 65 & $20(30.8)$ & $10(50.0)$ \\
\hline$>500$ & 26 & $9(34.6)$ & $2(22.2)$ \\
\hline Number of ROS1 samples tested last year? $(n=601)^{\mathrm{e}}$ & 601 & $256(42.6)^{*}$ & $118(46.1)$ \\
\hline No clinical samples tested & 32 & $18(56.3)$ & $6(33.3)$ \\
\hline$<10$ & 37 & $18(48.6)$ & $8(44.4)$ \\
\hline $10-99$ & 197 & $83(42.1)$ & $40(48.2)$ \\
\hline $100-249$ & 128 & $54(42.2)$ & $27(50)$ \\
\hline $250-499$ & 126 & $52(41.3)$ & $21(40.4)$ \\
\hline$>500$ & 81 & $31(38.3)$ & $16(51.6)$ \\
\hline Number of $A L K$ samples tested last year? $(n=1193)^{\mathrm{e}}$ & 1193 & $450(37.7)$ & $188(41.8)$ \\
\hline No clinical samples tested & 23 & $9(39.1)$ & $4(44.4)$ \\
\hline$<10$ & 25 & $9(36.0)$ & $1(11.1)$ \\
\hline $10-99$ & 333 & $118(35.4)$ & $47(39.8)$ \\
\hline $100-249$ & 374 & $142(37.9)$ & $66(46.5)$ \\
\hline $250-499$ & 281 & $113(40.2)$ & $41(36.3)$ \\
\hline$>500$ & 157 & $59(37.6)$ & $29(49.2)$ \\
\hline Number of $P D-L 1$ samples tested last year? $(n=491)^{\mathrm{e}}$ & 491 & $258(52.5)$ & $102(39.5)$ \\
\hline No clinical samples tested & 23 & $9(39.1)$ & $3(33.3)$ \\
\hline$<10$ & 29 & $18(62.1)$ & $8(44.4)$ \\
\hline $10-99$ & 193 & $96(49.7)$ & $33(34.4)$ \\
\hline $100-249$ & 117 & $66(56.4)$ & $32(48.5)$ \\
\hline $250-499$ & 90 & $47(52.2)$ & $16(34.0)$ \\
\hline$>500$ & 39 & $22(56.4)$ & $10(45.5)$ \\
\hline Number of KRAS samples tested last year? $(n=221)^{\mathrm{e}}$ & 221 & $92(41.6)^{*}$ & $39(42.4)$ \\
\hline No clinical samples tested & 6 & $5(83.3)$ & $2(40.0)$ \\
\hline$<10$ & 5 & $3(60.0)$ & $0(0.0)$ \\
\hline $10-99$ & 43 & $21(48.8)$ & $9(42.9)$ \\
\hline $100-249$ & 91 & $34(37.4)$ & $13(38.2)$ \\
\hline $250-499$ & 53 & $22(41.5)$ & $12(54.5)$ \\
\hline$>500$ & 23 & $7(30.4)$ & $3(42.9)$ \\
\hline Number of NRAS samples tested last year? $(n=219)^{\mathrm{e}}$ & 219 & $90(41.1)^{*}$ & $39(43.3)$ \\
\hline No clinical samples tested & 7 & $6(85.7)$ & $2(33.3)$ \\
\hline$<10$ & 4 & $2(50.0)$ & $0(0.0)$ \\
\hline $10-99$ & 60 & $31(51.7)$ & $16(51.6)$ \\
\hline $100-249$ & 90 & $31(34.4)$ & $10(32.3)$ \\
\hline $250-499$ & 44 & $14(31.8)$ & $8(57.1)$ \\
\hline$>500$ & 14 & $6(42.9)$ & $3(50.0)$ \\
\hline Number of $B R A F$ samples tested last year? $(n=207)^{\mathrm{e}}$ & 207 & $84(40.6)$ & $37(44.0)$ \\
\hline No clinical samples tested & 10 & $8(80.0)$ & $5(62.5)$ \\
\hline$<10$ & 18 & $4(22.2)$ & $1(25.0)$ \\
\hline
\end{tabular}


Table 2 (continued)

\begin{tabular}{llll}
\hline Laboratory characteristics & $N$ & $\begin{array}{l}\text { Number of laboratories who received the survey } \\
\text { (min 1 error in EQA scheme) (\%) }\end{array}$ & $\begin{array}{l}\text { Number of laboratories who responded } \\
\text { (\% of participants receiving the survey) }\end{array}$ \\
\hline $10-99$ & 73 & $30(41.1)$ & $15(50.0)$ \\
$100-249$ & 65 & $26(40.0)$ & $8(30.8)$ \\
$250-499$ & 31 & $12(38.7)$ & $6(50.0)$ \\
$>500$ & 10 & $4(40.0)$ & $2(50.0)$ \\
\hline
\end{tabular}

${ }^{*} p<0.05$. Exact $p$ values and odds ratios are shown in Supplemental Data $2 \mathrm{~A}$

a "Industry" laboratories are those developing diagnostic commercial kits. (Private) laboratories are not within a hospital's infrastructure. Hospital laboratories included laboratories in private and public hospitals. University and research included education and research hospitals, university hospitals, university laboratories, and anti-cancer centres [14]

${ }^{b}$ Laboratories under the department of pathology are those performing pathology review and the analytical tests in the same department

${ }^{\mathrm{c}}$ Accreditation is defined as compliant to ISO 15189 [15] or relevant national standards, such as CAP 15189 [16]

${ }^{\mathrm{d}}$ Laboratories who outsourced the analysis sent the samples to another laboratory for any part of the TTP, being either neoplastic cell percentage estimation, DNA extraction, or the genomic analysis

${ }^{\mathrm{e}}$ The number of staff involved in the TTP and annual samples tested were used a measure of the size and experience of the laboratory

$A L K$, ALK receptor tyrosine kinase; $B R A F$, B-Raf proto-oncogene; $E G F R$, epidermal growth factor receptor; $E Q A$, external quality assessment; $K R A S$, KRAS proto-oncogene; $N$, number; $N R A S$, NRAS proto-oncogene; $P D-L 1$, programmed death ligand 1; ROS1, ROS proto-oncogene 1; TTP, total test process

received sample material as the cause. On the other hand, they more frequently reported reagent problems. Accredited laboratories less frequently encountered reagent problems.

Laboratories with a larger staff number (usually larger laboratories) had a reduced probability of encountering method-related problems. Testing more samples annually increased the chance of a personnel error to occur. Respondents who changed their testing method in the last 12 months prior to the survey were significantly more likely to obtain a problem with that methodology compared to laboratories who did not change anything to their methodology in this period. There was no significant relationship between any of the other causes and the laboratory characteristics (Supplemental Data 2C).

\section{Detection of errors during the EQA scheme}

Post-analytical problems were more likely to be detected after release of the EQA results especially for clerical and interpretation errors (Table 5). On the other hand, pre-analytical and analytical issues, such as equipment/technical or methodological problems and issues with the EQA material, were more likely to be picked up in advance (Table 5).

Laboratories with an error in the pre-analytical phase were more likely to encounter an analysis failure in the scheme. Laboratories with analytical problems more often obtained lower performance scores, and those with post-analytical problems had a significantly higher score, due to the occurrence of fewer technical failures. More specifically, personnel errors, equipment, and reagent problems lowered the score in the EQA scheme, while laboratories reporting a problem with the material were more likely to obtain a technical failure. Exact $p$ values and ORs are shown in Supplemental Data 2C.
The EQA participants undertook specific corrective actions, which were significantly linked to the time in the TPP and cause (Supplemental Fig. 1). Respondents with a personnel error more often had an analysis error in the subsequent EQA scheme, but there was no effect by any other error cause on the performance criteria in the next EQA scheme [24].

\section{Discussion}

Several studies have evaluated the longitudinal improvement of biomarker testing in NSCLC and mCRC for different laboratories, samples, and methods [4-13]. Even though error rates are published [4-13] and some providers request root cause analyses, no information is yet available on the underlying causes for deviating EQA results in the laboratories for molecular oncology.

\section{Response to root cause surveys}

Our data on root causes of deviating EQA results demonstrated that laboratories who are accredited or test more samples annually (for ROS1, KRAS and NRAS) were less likely to receive the survey. Keeping in mind that the surveys were sent only to participants with deviating results, these findings are not surprising. It has been described that accredited laboratories testing more samples demonstrated a better performance in the EQA schemes [14]. In contrast, laboratories that outsourced (a part of) their analysis reported more EQA errors. ISO 15189 states that the laboratory shall have a documented procedure for selecting and evaluating referral laboratories, and is responsible for monitoring their quality [15]. More investigations are needed on which elements of the 
Table 3 Time point of deviating EQA results in the different subschemes

\begin{tabular}{|c|c|c|c|c|c|c|c|}
\hline & & & \multirow[b]{2}{*}{$N($ total $=514)$} & \multicolumn{4}{|c|}{ Error phase $(\%)$} \\
\hline & & & & $\begin{array}{l}\text { Pre- } \\
\text { analytical }\end{array}$ & Analytical & $\begin{array}{l}\text { Post- } \\
\text { analytical }\end{array}$ & Unknown \\
\hline NSCLC & Technique & Marker & 424 & 15.3 & 30.2 & 48.1 & 6.4 \\
\hline \multirow[t]{3}{*}{ Digital cases } & FISH (interpretation only) & $A L K$ & 7 & 0.0 & 0.0 & 100.0 & 0.0 \\
\hline & & ROS1 & 33 & 3.0 & 3.0 & 87.9 & 6.1 \\
\hline & IHC (interpretation only) & PD-L1 & 30 & 6.7 & 3.3 & 83.3 & 6.7 \\
\hline \multirow[t]{11}{*}{ FFPE samples } & FISH & $A L K$ & 25 & 44.0 & 20.0 & 28.0 & 8.0 \\
\hline & & ROS1 & 49 & 30.6 & 18.4 & 36.7 & 14.3 \\
\hline & IHC & ALK & 31 & 16.1 & 38.7 & 41.9 & 3.2 \\
\hline & & ROS1 & 17 & 5.9 & 35.3 & 52.9 & 5.9 \\
\hline & & PD-L1 & 63 & 19.0 & 12.7 & 55.6 & 12.7 \\
\hline & IHC (technical assessment) & ALK & 36 & 13.9 & 80.6 & 5.6 & 0.0 \\
\hline & & ROS1 & 6 & 16.7 & 50.0 & 33.3 & 0.0 \\
\hline & & PD-L1 & 3 & 33.3 & 33.3 & 0.0 & 33.3 \\
\hline & Variant analysis & $E G F R$ & 108 & 10.2 & 39.8 & 47.2 & 2.8 \\
\hline & & $K R A S$ & 13 & 0.0 & 53.8 & 46.2 & 0.0 \\
\hline & & $B R A F$ & 3 & 0.0 & 100.0 & 0.0 & 0.0 \\
\hline mCRC & Technique & Marker & 90 & 30.0 & 42.2 & 24.4 & 3.3 \\
\hline \multirow[t]{5}{*}{ FFPE samples } & Variant analysis & $K R A S$ & 53 & 28.3 & 47.2 & 18.9 & 5.7 \\
\hline & & $N R A S$ & 12 & 25.0 & 33.3 & 41.7 & 0.0 \\
\hline & & $B R A F$ & 10 & 30.0 & 30.0 & 40.0 & 0.0 \\
\hline & & WT & 10 & 40.0 & 50.0 & 10.0 & 0.0 \\
\hline & & No neoplastic cells & 5 & 40.0 & 20.0 & 40.0 & 0.0 \\
\hline
\end{tabular}

For $A L K$ and ROS1 analysis, participation to the FISH subschemes automatically enrolled the laboratory for interpretation of digital FISH cases besides the FFPE cases [12]. Expression of programmed death ligand $1(P D-L 1)$ was assessed (since 2017) by providing FFPE samples for IHC and digital cases for interpretation of the IHC stain. For mCRC EQA schemes, one of the provided colon cases included a case without neoplastic cells to verify the testing practice of the participants in this case. The most frequent reported time points of occurrence are italicized. Start and endpoints of phases in this study were defined based on definitions in ISO 15189 (clauses 3.14 and 3.15) [15]. The pre-analytical phase was communicated in the survey as the time from sample reception until selection and estimation of the neoplastic cell percentage during pathologist review (for variant analysis) and until sample pretreatment (for FISH or IHC). The analytical phase started from DNA extraction (if applicable) and the actual biomarker test, i.e., all steps of mutation analysis, gene rearrangement, or IHC analysis according to the pre-determined protocol. The post-analytical phase occurred between the readout of the analytical results (interpretation of mutation analysis curves, of the staining intensity/pattern, or reading of the split/single FISH nuclei), and reporting of the results, in this case when entering the results in the electronic EQA datasheets. $A L K$, ALK receptor tyrosine kinase; $B R A F$, B-Raf proto-oncogene; $E G F R$, epidermal growth factor receptor; $E Q A$, external quality assessment; FFPE, formalin-fixed paraffin embedded; FISH, fluorescence in situ hybridization; IHC, immunohistochemistry; KRAS, KRAS proto-oncogene; $m C R C$, metastatic colorectal cancer; $N$, number; $N R A S$, NRAS protooncogene; NSCLC, non-small cell lung cancer; $P D-L 1$, programmed death ligand 1; ROS1, ROS proto-oncogene 1; WT, wild-type

TTP are being outsourced in routine, the structure of laboratory networks, and how high quality is ensured.

Accredited laboratories were also more likely to reply to the survey. Participation to quality improvement projects such as survey completion or workshop attendance [25] has previously shown to increase EQA performance in mCRC and might contribute to the better performance for accredited participants. We acknowledge that not all countries have responded, and error causes might shift when taking into account data from nonrespondents. Nevertheless, with data from 185 laboratories worldwide which encompassed $44.0 \%$ of the incorrect samples, this is a valuable first assessment of causes underlying deviating EQA results. The uniform taxonomy and participant-tailored surveys allowed to compare the results between the different survey rounds. A continued follow-up might be useful to evaluate if the conclusions are still valid when evaluating more respondents, as well as for other predictive markers currently not included in the schemes.

\section{Time point in the total test process and cause of deviating EQA results}

The causes of deviating EQA outcomes were related to the indication (NSCLC or mCRC) and included subschemes. It must be noted that for the FFPE samples, more interpretation problems were reported for ROS1 compared to $A L K$, even when tested by the same technique type (FISH or IHC) and even more so for PD-L1 IHC (Table 4). This is consistent with 


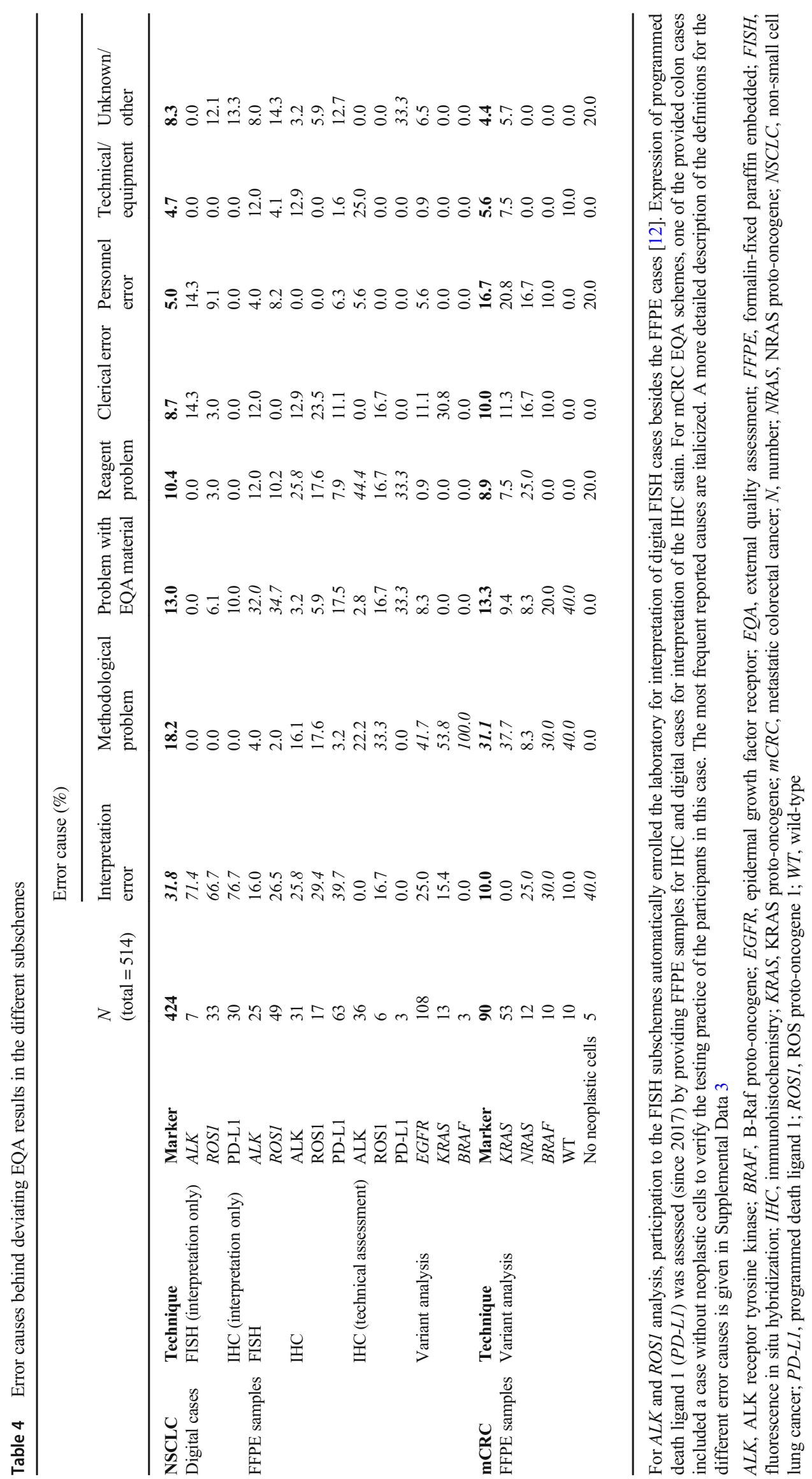


Table 5 Error phase and cause related to laboratory characteristics and EQA scheme performance

\begin{tabular}{|c|c|c|c|c|c|c|c|c|c|}
\hline Error phas & & & Error cau & & & & & & \\
\hline $\begin{array}{l}\text { Pre- } \\
\text { analytical }\end{array}$ & Analytical & $\begin{array}{l}\text { Post- } \\
\text { analytical }\end{array}$ & $\begin{array}{l}\text { Clerical } \\
\text { error }\end{array}$ & $\begin{array}{l}\text { Equipment/ } \\
\text { technical } \\
\text { problem }\end{array}$ & $\begin{array}{l}\text { Interpretation } \\
\text { error }\end{array}$ & $\begin{array}{l}\text { Methodological } \\
\text { problem }\end{array}$ & $\begin{array}{l}\text { Personnel } \\
\text { error }\end{array}$ & $\begin{array}{l}\text { Problem } \\
\text { with } \\
\text { EQA } \\
\text { material }\end{array}$ & $\begin{array}{l}\text { Reagent } \\
\text { problem }\end{array}$ \\
\hline
\end{tabular}

\begin{tabular}{|c|c|c|c|c|c|c|c|c|c|c|}
\hline \multicolumn{11}{|c|}{ Laboratory characteristics } \\
\hline Setting & $\leftrightarrow$ & $\leftrightarrow$ & $\leftrightarrow$ & $\leftrightarrow$ & $\leftrightarrow$ & $\leftrightarrow$ & $\leftrightarrow$ & $\leftrightarrow$ & $\leftrightarrow$ & $\leftrightarrow$ \\
\hline Accreditation & $\leftrightarrow$ & $\leftrightarrow$ & $\leftrightarrow$ & $\leftrightarrow$ & $\leftrightarrow$ & $\leftrightarrow$ & $\leftrightarrow$ & $\leftrightarrow$ & $\leftrightarrow$ & $\downarrow * *$ \\
\hline $\begin{array}{l}\text { Higher nr. of staff } \\
\text { involved in } \\
\text { biomarker test }\end{array}$ & $\leftrightarrow$ & $\leftrightarrow$ & $\leftrightarrow$ & $\leftrightarrow$ & $\leftrightarrow$ & $\leftrightarrow$ & $\downarrow * *$ & $\leftrightarrow$ & $\leftrightarrow$ & $\leftrightarrow$ \\
\hline $\begin{array}{l}\text { Analysis under dept. } \\
\text { of pathology }\end{array}$ & $\downarrow * *$ & $\leftrightarrow$ & $\leftrightarrow$ & $\leftrightarrow$ & $\leftrightarrow$ & $\leftrightarrow$ & $\leftrightarrow$ & $\leftrightarrow$ & $\downarrow^{* * * *}$ & $\uparrow *$ \\
\hline $\begin{array}{l}\text { Higher nr. of } \\
\text { samples tested per } \\
\text { year }\end{array}$ & $\leftrightarrow$ & $\leftrightarrow$ & $\leftrightarrow$ & $\leftrightarrow$ & $\leftrightarrow$ & $\leftrightarrow$ & $\leftrightarrow$ & $\uparrow *$ & $\leftrightarrow$ & $\leftrightarrow$ \\
\hline $\begin{array}{l}\text { Change in test } \\
\text { methodology in } \\
\text { last } 12 \text { months }\end{array}$ & $\leftrightarrow$ & $\leftrightarrow$ & $\leftrightarrow$ & $\leftrightarrow$ & $\leftrightarrow$ & $\leftrightarrow$ & $\uparrow *$ & $\leftrightarrow$ & $\leftrightarrow$ & $\leftrightarrow$ \\
\hline Methodology type & $\leftrightarrow$ & $\leftrightarrow$ & $\leftrightarrow$ & $\leftrightarrow$ & $\leftrightarrow$ & $\leftrightarrow$ & $\leftrightarrow$ & $\leftrightarrow$ & $\leftrightarrow$ & $\leftrightarrow$ \\
\hline \multicolumn{11}{|l|}{ EQA performance } \\
\hline $\begin{array}{l}\text { Laboratories who } \\
\text { detected the error } \\
\text { after release of } \\
\text { EQA results }\end{array}$ & $\downarrow^{*}$ & $\downarrow * *$ & $\uparrow * * *$ & $\uparrow *$ & $\downarrow * *$ & $\uparrow * * *$ & $\downarrow^{*}$ & $\leftrightarrow$ & $\downarrow * * *$ & $\leftrightarrow$ \\
\hline $\begin{array}{l}\text { Laboratories with a } \\
\text { higher } \\
\text { performance score }\end{array}$ & $\leftrightarrow$ & $\downarrow * * *$ & $\uparrow * * * *$ & $\leftrightarrow$ & $\downarrow^{* *}$ & $\uparrow * * *$ & $\leftrightarrow$ & $\downarrow^{* *}$ & $\uparrow * * *$ & $\downarrow * * *$ \\
\hline $\begin{array}{l}\text { Laboratories who } \\
\text { were successful }\end{array}$ & $\leftrightarrow$ & $\leftrightarrow$ & $\leftrightarrow$ & $\leftrightarrow$ & $\leftrightarrow$ & $\leftrightarrow$ & $\leftrightarrow$ & $\leftrightarrow$ & $\leftrightarrow$ & $\leftrightarrow$ \\
\hline $\begin{array}{l}\text { Laboratories who } \\
\text { obtained fewer } \\
\text { genotyping errors }\end{array}$ & $\leftrightarrow$ & $\leftrightarrow$ & $\leftrightarrow$ & $\leftrightarrow$ & $\leftrightarrow$ & $\leftrightarrow$ & $\leftrightarrow$ & $\downarrow * * *$ & $\uparrow * * * *$ & $\leftrightarrow$ \\
\hline $\begin{array}{l}\text { Laboratories who } \\
\text { obtained fewer } \\
\text { analysis failures }\end{array}$ & $\downarrow * *$ & $\leftrightarrow$ & $\uparrow *$ & $\leftrightarrow$ & $\leftrightarrow$ & $\uparrow *$ & $\leftrightarrow$ & $\leftrightarrow$ & $\downarrow^{* *}$ & $\leftrightarrow$ \\
\hline
\end{tabular}

$\downarrow$ Statistical decrease in error phase/cause, $\uparrow$ statistical increase in error phase/cause, $\leftrightarrow$ no statistical effect observed. $* p<0.05, * * p<0.01, * * * p<0.001$. Detailed $p$ values and odds ratios are given in Supplemental Data 2C

Start and endpoints of phases in this study were defined based on definitions in ISO 15189 (clauses 3.14 and 3.15) [15]. The pre-analytical phase was communicated in the survey as the time from sample reception until selection and estimation of the neoplastic cell percentage during pathologist review (for variant analysis) and until sample pre-treatment (for FISH or IHC). The analytical phase started from DNA extraction (if applicable) and the actual biomarker test, i.e., all steps of mutation analysis, gene rearrangement, or IHC analysis according to the pre-determined protocol. The post-analytical phase occurred between the readout of the analytical results (interpretation of mutation analysis curves, of the staining intensity/pattern, or reading of the split/single FISH nuclei), and reporting of the results, in this case when entering the results in the electronic EQA datasheets. Laboratories under the department of pathology are those performing pathology review and the analytical tests in the same department. Accreditation is defined as compliant to ISO 15189 [15] or relevant national standards, such as CAP 15189 [16]. The number of staff involved and annual samples tested were used a measure of the size and experience of the laboratory [24]. A change in method represents laboratories who changed their analysis method or protocol in the last 12 months prior to the survey. A more detailed description of the definitions for the different error causes is given in Supplemental Data 3. EQA, external quality assessment; $n r$., number

previously reported increased error rates for ROS1 compared to $A L K$, explained by an increased experience with $A L K$, as $R O S 1$ testing was only approved since 2016 [12]. In the survey period, fewer guidelines were thus available for ROS1 interpretation, and no Food and Drug Administration-approved companion diagnostic (which was the case for $A L K$ ). For $P D-L 1$, a similar assumption can be made as it is only recently required for testing and its interpretation poses additional challenges due the availability of different commercially antibodies with varying cut-offs for positivity for different therapies [26].

In case sample problems were reported for FISH, the most prominent reasons were suboptimal sample quality $(20.9 \%)$ or too few neoplastic cells (14.9\%) (Supplemental Data 3). Estimation of the neoplastic cell content in EQA schemes 
has been reported as highly variable [27]. Nevertheless, materials were carefully validated beforehand to have sufficient neoplastic cells and lacking tumor heterogeneity, and other peers were able to successfully analyze them. Even though digital FISH cases only assess the post-analytical phase, for two cases, the survey respondents mentioned a problem during creation of the images at the pre- or analytical phase to be at the basis of the interpretation error (Table 3).

For variant analysis, the laboratories frequently reported the lack of a specific variant in the analysis method (Supplemental Data 3), especially for mCRC (17.8\%) compared to NSCLC $(5.0 \%)$. This is a well-known problem, as in 2013, the drug label for cetuximab and panitumumab was extended to include codons $12,13,59,61,117$, and 146 for both the KRAS and NRAS genes, but not all laboratories have adapted their testing strategy [11]. Also, insufficient method sensitivity was reported, as well as misinterpretation of obtained sequencing curves (e.g., results around the threshold), which are especially important in routine for variants at low frequencies such as EGFR c.2369C $>$ T p.(Thr790Met) LRG_304p1. The number of errors reported in wild-type cases was too low to make solid assumptions.

The specific causes suggest that EQA providers could benefit from requesting root cause analyses after the schemes to provide more tailored education to participants. For instance, the provision of digital or paper-based cases to assess interpretation or variant classifications could aid in the interpretation for specific markers. Given the broad variety of methodologies used by the participants completing the survey, the performance of these methods might have further contributed to the error causes. Indeed, different performances have been reported depending on the applied PD-L1 IHC clones (personal observations), ALK IHC clones, or EGFR variant analysis method in the same ESP NSCLC EQA schemes, and depending on $R A S$ analysis methods in the ESP mCRC EQA schemes [11, 13, 28]. Challenging samples might be included (albeit educational) with rare variants to assess the inclusion of all relevant mutations or their detection at low allele frequencies. Schemes should thus be fit for purpose [19] and should cover the entire examination process as required by ISO 15189 [15]. As the samples in the EQA scheme were pre-cut and labeled, several pre-analytical steps were outside the study scope. Research on routine cases is advised to assess problems during sample embedding, cutting, or labelling.

\section{Error causes for the different laboratory characteristics}

Previous longitudinal results indicated that experience (by accreditation, a research setting, or testing more annual samples) positively affected EQA scores [14]. Our findings revealed that personnel errors increased when testing more samples, probably due to the increased work pressure. Laboratory automation might be the way forward to reduce these errors. Also, laboratories with an increased number of staff had fewer method-based errors, by the probable larger capacity of professionally trained personnel to perform a certain method [29]. Accredited laboratories less frequently had a reagent problem, possibly due to working according to standard operating procedures. As these reagent problems significantly lowered the EQA performance, this might explain their previously better performance.

Our data also revealed that laboratories operating under the department of pathology less often reported sample-related issues (Table 3), but more frequently encountered reagent problems, as they were more frequently involved in IHC analysis compared to molecular laboratories. The positive influence of pathology review in decreasing specimen problems in this study stresses its importance to obtain accurate results further downstream the TTP.

We did not observe a difference in error rates concerning the method type (i.e., NGS versus pyrosequencing), in agreement with previous studies [14]. However, we observed that a change in test method during the last year resulted in significantly more method-related error causes, highlighting the importance of test validation before implementation in clinical practice.

\section{Detection of errors during the EQA scheme}

Post-analytical clerical and interpretation problems were less likely detected before release of the results (Table 5) in contrast to equipment, methodological, and sample-related problems. This seems logical, given that post-analytical issues occur closer to reporting of the results and have less time to be picked up by a quality control step. This might explain the higher error rates previously reported for ROS1 compared to $A L K$ [12], as this marker now indeed revealed a large fraction of clerical and interpretation causes.

Looking at the current scheme performance (Table 5), errors in the pre-analytical phase were more prominent for participants with lower performance scores and more technical failures. This again underlines the importance of pre-analytic quality control to prevent technical failures resulting from selecting insufficient neoplastic cells [27].

None of the causes had a significant effect on future scheme performances except for personnel errors. In this case, laboratories most frequently responded by retraining their staff [24]. Also, for the majority of errors, an appropriate corrective action was undertaken (Supplemental figure 1).

\section{Conclusions}

To conclude, causes of deviating EQA results were indication, marker, and technique dependent. The phase and underlying 
cause differently affected the EQA performance, either by an increase in test failures or false-positive/false-negative results. Our findings advocate using surveys by EQA providers to specifically tailor the schemes for set-up, feedback, and offered sample types. Timely quality checks aid to uncover deviating results and should be additionally implemented in the post-analytical phase as these errors were often not identified in the laboratory. Accredited laboratories were more likely to respond and had fewer reagent problems, which could explain their previously reported better performance. We detected an important effect of pathology review to reduce technical failures and of protocol changes to increase method-related problems.

Acknowledgments This project would not have been possible without the support of the following people:

- The European Society of Pathology for the joint organization of the EQA schemes and administrative support

- The EQA steering committee, scheme assessors, medical and technical experts, and laboratories or regional scheme organizers involved in validation and preparation of the EQA scheme samples

- All laboratories that participated to the ESP Lung EQA schemes between 2015 and 2018 and the ESP Colon EQA schemes between 2016 and 2018 and completed the survey

- Colleagues of the BQA Research Unit for the coordination and administrative support of the EQA schemes

- Yann Van Royen, for his assistance in creation of the surveys and collection of the data during the 2017 and 2018 ESP Lung EQA schemes

- Annouschka Laenen, Leuven Biostatistics and Statistical Bioinformatics Centre (L-BioStat) and the Leuven Cancer Institute for performing the statistical analyses

Author contributions $\mathrm{CK}$ and EMCD conceived and designed the study set-up and were responsible for creation and sending of the surveys, data collection, and harmonization. $\mathrm{CK}$ and EMCD were responsible for the statistical analysis. ES provided technical expertise during the EQA schemes. All authors were involved in writing of the manuscript.

Funding A research grant from Amgen Inc., Thousand Oaks, CA 91320, USA (grant number not applicable) was obtained for this study during the 2016 and 2017 ESP Colon EQA schemes. An unrestricted research grant (grant number not applicable) from Pfizer Oncology was obtained for the organization of the ESP Lung EQA schemes, irrespective of the performed research.

Data availability The datasets generated during and/or analyzed during the current study are available from the corresponding author on reasonable request.

\section{Compliance with ethical standards}

Conflict of interest $\mathrm{CK}$ and EMCD have nothing to declare. ES received honoraria for consultancy/advisory board from AstraZeneca, MSD/ Merck, Roche, Pfizer, Bayer, Novartis, BMS, BioRad, Illumina, Ageno BioSciences, Janssen Cilag (Johnson\&Johnson), and BioCartis; speaker's fee from AstraZeneca, Ageno BioSciences, Roche, Pfizer, Novartis, BioRad, Illumina, and BioCartis; and (unrestricted) grants from Boehringer Ingelheim, BMS, Biocartis, BioRad, Ageno BioSciences, and Roche (all outside the submitted work and fees to UMCG).

Ethics The samples originated from tissue blocks of leftover patient material obtained during routine care. Each scheme organizer signed a subcontractor agreement stating that the way in which the samples were obtained conformed to the national legal requirements for the use of patient samples. The samples were excluded from research regulations requiring informed consent.

Open Access This article is licensed under a Creative Commons Attribution 4.0 International License, which permits use, sharing, adaptation, distribution and reproduction in any medium or format, as long as you give appropriate credit to the original author(s) and the source, provide a link to the Creative Commons licence, and indicate if changes were made. The images or other third party material in this article are included in the article's Creative Commons licence, unless indicated otherwise in a credit line to the material. If material is not included in the article's Creative Commons licence and your intended use is not permitted by statutory regulation or exceeds the permitted use, you will need to obtain permission directly from the copyright holder. To view a copy of this licence, visit http://creativecommons.org/licenses/by/4.0/.

\section{References}

1. Lindeman NI, Cagle PT, Beasley M et al (2013) Molecular testing guideline for selection of lung cancer patients for EGFR and ALK tyrosine kinase inhibitors: guideline from the College of American Pathologists, International Association for the Study of Lung Cancer, and Association for Molecular Pathology. J Thorac Oncol 8(7):823-859. https://doi.org/10.1097/JTO.0b013e318290868f

2. Lindeman NI, Cagle PT, Aisner DL, Arcila ME, Beasley MB, Bernicker EH, Colasacco C, Dacic S, Hirsch FR, Kerr K, Kwiatkowski DJ, Ladanyi M, Nowak JA, Sholl L, TempleSmolkin R, Solomon B, Souter LH, Thunnissen E, Tsao MS, Ventura CB, Wynes MW, Yatabe Y (2018) Updated molecular testing guideline for the selection of lung cancer patients for treatment with targeted tyrosine kinase inhibitors: guideline from the College of American Pathologists, the International Association for the Study of Lung Cancer, and the Association for Molecular Pathology. J Mol Diagn 20(2):129-159. https://doi.org/10.1016/j. jmoldx.2017.11.004

3. Cree IA, Deans ZC, Ligtenberg MJL, Normanno N, Edsjö A, Rouleau E, Solé F, Thunnissen E, Timens W, Schuuring E, Dequeker E, Murray S, Dietel M, Groenen P, van Krieken J, European Society of Pathology Task Force on Quality Assurance in Molecular Pathology., Royal College of Pathologists (2014) Guidance for laboratories performing molecular pathology for cancer patients. J Clin Pathol 67(11):923-931. https://doi.org/10.1136/ jclinpath-2014-202404

4. Patton S, Normanno N, Blackhall F, Murray S, Kerr KM, Dietel M, Filipits M, Benlloch S, Popat S, Stahel R, Thunnissen E (2014) Assessing standardization of molecular testing for non-small-cell lung cancer: results of a worldwide external quality assessment (EQA) scheme for EGFR mutation testing. Br J Cancer 111(2): 413-420. https://doi.org/10.1038/bjc.2014.353

5. Dequeker EMC, Keppens C, Egele C, Delen S, Lamy A, Lemoine A, Sabourin JC, Andrieu C, Ligtenberg M, Fetique D, Tops B, Descarpentries C, Blons H, Denoux Y, Aube C, Penault-Llorca F, Hofman P, Leroy K, le Marechal C, Doucet L, Duranton-Tanneur 
V, Pedeutour F, Soubeyran I, Côté JF, Emile JF, Vignaud JM, Monhoven N, Haddad V, Laurent-Puig P, van Krieken H, Nowak F, Lonchamp E, Bellocq JP, Rouleau E (2016) Three rounds of external quality assessment in France to evaluate the performance of 28 platforms for multiparametric molecular testing in metastatic colorectal and non-small cell lung cancer. J Mol Diagn 18(2):205214. https://doi.org/10.1016/j.jmoldx.2015.09.004

6. Normanno N, Fenizia F, Castiglione F, Barberis M, Taddei GL, Truini M, De Rosa G, Pinto C, Marchetti A (2017) External quality assessment for EGFR mutations in Italy: improvements in performances over the time. ESMO Open 2(2):e000160. https://doi.org/ 10.1136/esmoopen-2017-000160

7. Richman SD, Fairley J, Butler R, Deans ZC (2017) RAS screening in colorectal cancer: a comprehensive analysis of the results from the UK NEQAS colorectal cancer external quality assurance schemes (2009-2016). Virchows Arch 471(6):721-729. https:// doi.org/10.1007/s00428-017-2162-7

8. Normanno N, Pinto C, Castiglione F, Fenizia F, Barberis M, Marchetti A, Fontanini G, De Rosa G, Taddei GL (2015) The Italian external quality assessment for RAS testing in colorectal carcinoma identifies methods-related inter-laboratory differences. J Transl Med 13:287. https://doi.org/10.1186/s12967-015-0655-1

9. Marchetti A, Barberis M, Papotti M, Rossi G, Franco R, Malatesta S, Buttitta F, Ardizzoni A, Crinò L, Gridelli C, Taddei GL, Clemente C, Scagliotti G, Normanno N, Pinto C (2014) ALK rearrangement testing by FISH analysis in non-small-cell lung cancer patients: results of the first italian external quality assurance scheme. J Thorac Oncol 9(10):1470-1476. https://doi.org/10. 1097/JTO.0000000000000280

10. Deans ZC, Bilbe N, O'Sullivan B, Lazarou LP, de Castro DG, Parry S, Dodson A, Taniere P, Clark C, Butler R (2013) Improvement in the quality of molecular analysis of EGFR in non-small-cell lung cancer detected by three rounds of external quality assessment. J Clin Pathol 66(4):319-325. https://doi.org/10.1136/jclinpath-2012-201227

11. Dufraing K, Keppens C, Tack V, Siebers AG, Kafatos G, Dube S, Demonty G, Lowe K, Kroeze LI, Ligtenberg MJL, Normanno N, Tembuyser L, Sara VB, van Krieken JH, C Dequeker EM (2020) Evolution of RAS testing over time: factors influencing mutation rates in metastatic colorectal cancer patients. Colorect Cancer 1(9): 1-17. https://doi.org/10.2217/crc-2019-0013

12. Keppens $\mathrm{C}$, Tack V, 't Hart $\mathrm{N}$ et al (2018) A stitch in time saves nine: external quality assessment rounds demonstrate improved quality of biomarker analysis in lung cancer. Oncotarget 9(29): 20524-20538. https://doi.org/10.18632/oncotarget.24980

13. Keppens C, Dequeker EMC, Rouleau E, 't Hart N, Bubendorf L, Dufraing K, Garrec C, Guéguen P, Lamy A, Marchetti A, Pauwels $\mathrm{P}$, Ryska A, Tack V, Tornillo L, van Casteren K, von der Thüsen JH, Zwaenepoel K, Lissenberg-Witte B, Thunnissen E, Schuuring E (2020) Sensitive detection methods are key to identify secondary EGFR c.2369C $>$ T p.(Thr790Met) in non-small cell lung cancer tissue samples. BMC Cancer 20(1):366. https://doi.org/10.1186/ s12885-020-06831-3

14. Tack V, Schuuring E, Keppens C, 't Hart N, Pauwels P, van Krieken H, Dequeker EMC (2018) Accreditation, setting and experience as indicators to assure quality in oncology biomarker testing laboratories. Br J Cancer 119(5):605-614. https://doi.org/10. 1038/s41416-018-0204-9

15. International Organization for Standardization (ISO) (2012) ISO 15189:2012 medical laboratories - particular requirements for quality and competence. ISO, Geneva

16. Clinical Laboratory Improvement Amendments of 1988, 42 U.S.C. 263a PL100-578 (1988) Laboratory requirements, 2003, 42 C.F.R. Chapter IV, Part 493

17. International Organization for Standardization (ISO) (2010) ISO 17043:2010 conformity assessment - general requirements for proficiency testing. ISO, Geneva
18. van Krieken JH, Normanno N, Blackhall F, Boone E, Botti G, Carneiro F, Celik I, Ciardiello F, Cree IA, Deans ZC, Edsjö A, Groenen PJTA, Kamarainen O, Kreipe HH, Ligtenberg MJL, Marchetti A, Murray S, Opdam FJM, Patterson SD, Patton S, Pinto C, Rouleau E, Schuuring E, Sterck S, Taron M, Tejpar S, Timens W, Thunnissen E, van de Ven PM, Siebers AG, Dequeker E (2013) Guideline on the requirements of external quality assessment programs in molecular pathology. Virchows Arch 462(1):27-37. https://doi.org/10.1007/s00428-012-1354-4

19. Dufraing K, Fenizia F, Torlakovic E, Wolstenholme N, Deans ZC, Rouleau E, Vyberg M, Parry S, Schuuring E, Dequeker E, on behalf of IQNPath ABSL (2020) Biomarker testing in oncology - requirements for organizing external quality assessment programs to improve the performance of laboratory testing: revision of an expert opinion paper on behalf of IQNPath ABSL. Virchows Arch:1-13. https://doi.org/10.1007/s00428-020-02928-z

20. Hammerling A (2012) Review of medical errors in laboratory diagnostics and where we are today. Lab Med 43(2):41-44. https:// doi.org/10.1309/LM6ER9WJR1IHQAUY

21. Kloosterman A, Sjerps M, Quak A (2014) Error rates in forensic DNA analysis: definition, numbers, impact and communication. Forensic Sci Int Genet 12:77-85. https://doi.org/10.1016/j.fsigen. 2014.04.014

22. Kristensen GB, Meijer P (2017) Interpretation of EQA results and EQA-based trouble shooting. Biochem Med 27(1):49-62. https:// doi.org/10.11613/BM.2017.007

23. Sciacovelli L, Secchiero S, Padoan A, Plebani M (2018) External quality assessment programs in the context of ISO 15189 accreditation. Clin Chem Lab Med 56(10):1644-1654. https://doi.org/10. 1515/cclm-2017-1179

24. Keppens C, Schuuring E, Dequeker EM (2020) Managing deviating EQA results: a survey to assess the corrective and preventive actions of medical laboratories testing for oncological biomarkers. Diagnostics (Basel) 10(10):E837. https://doi.org/10.3390/ diagnostics10100837

25. Keppens C, Dufraing K, van Krieken HJ, Siebers AG, Kafatos G, Lowe K, Demonty G, Dequeker EMC (2019) European follow-up of incorrect biomarker results for colorectal cancer demonstrates the importance of quality improvement projects. Virchows Arch 475(1):25-37. https://doi.org/10.1007/s00428-019-02525-9

26. Büttner R, Gosney JR, Skov BG, Adam J, Motoi N, Bloom KJ, Dietel M, Longshore JW, López-Ríos F, Penault-Llorca F, Viale G, Wotherspoon AC, Kerr KM, Tsao MS (2017) Programmed deathligand 1 immunohistochemistry testing: a review of analytical assays and clinical implementation in non-small-cell lung cancer. J Clin Oncol 35(34):3867-3876. https://doi.org/10.1200/JCO.2017.74.7642

27. Dufraing K, De Hertogh G, Tack V, Keppens C, Dequeker EMC, van Krieken JH (2018) External quality assessment identifies training needs to determine the neoplastic cell content for biomarker testing. J Mol Diagn 20(4):455-464. https://doi.org/10.1016/j. jmoldx.2018.03.003

28. Keppens C, von der Thüsen JH, Pauwels P, Ryska A, 't Hart N, Schuuring E, Miller K, Thunnissen E, Zwaenepoel K, Dequeker EMC (2020) Staining performance of ALK and ROS1 immunohistochemistry and influence on molecular interpretation in NSCLC. J Mol Diagn 20:30482-30487. https://doi.org/10.1016/j.jmoldx. 2020.09.006

29. Jones BA, Darcy T, Souers RJ, Meier FA (2012) Staffing benchmarks for clinical laboratories: a College of American Pathologists Q-Probes study of laboratory staffing at 98 institutions. Arch Pathol Lab Med 136(2):140-147. https://doi.org/10.5858/arpa.20110206-CP

Publisher's note Springer Nature remains neutral with regard to jurisdictional claims in published maps and institutional affiliations. 